\title{
Massive integration of inorganic nanowire-based structures on solid substrates for device applications $\dagger$
}

\author{
Kwang Heo, ${ }^{a}$ Cheol-Joo Kim, ${ }^{b}$ Moon-Ho Jo ${ }^{* b}$ and Seunghun Hong $* a$ \\ Received 30th September 2008, Accepted 13th November 2008 \\ First published as an Advance Article on the web 6th January 2009 \\ DOI: $10.1039 / b 817136 j$
}

Inorganic nanowire-based devices have recently drawn extensive attention as one of the nextgeneration device architectures. Nevertheless, a lack of mass-production methods has been one of the major hurdles holding back the practical applications of such devices. Herein, we review three promising strategies for the massive assembly of inorganic nanowires for their device applications, which are topically selected: selective growth, selective assembly, and direct printing methods. The advantages and disadvantages of these methods are also discussed.

\subsection{Introduction}

As the conventional microelectronic industry approaches its technological and financial limitations, new advanced devices based on inorganic nanowires (NWs) have received extensive attention as one of the next-generation device architectures. However, their practical applications are hampered, mainly by difficulty in mass production of such devices. In conventional microfabrication processes, a semiconductor wafer is carved to fabricate desired circuit structures. However, in the case of NWbased devices, NWs are first synthesized on a solid substrate or in solution environments. Thus, it is required to selectively grow NWs for their sizes, positions and directions or assemble pregrown NWs onto the specific regions of solid substrates to build large-scale integrated devices, which is not an easy task.

${ }^{a}$ Department of Physics and Astronomy, Seoul National University, Kwanak-GuShillim-Dong, Seoul, Korea.E-mail: shong@phya.snu.ac.kr; Fax: +82-2-884-3002; Tel: +82-2-880-1343

${ }^{b}$ Department of Materials Science and Engineering, Pohang University of Science and Technology (POSTECH), Hyoja-Dong, Nam-Gu, Pohang, Gyungbuk, Korea. E-mail: mhjo@postech.ac.kr; Fax: +82-54-279-2158; Tel: $+82-54-279-2399$

$\uparrow$ This paper is part of a Journal of Materials Chemistry theme issue on Nanotubes and Nanowires. Guest editor: Z. L. Wang.
Here, we review three successful strategies to mass-produce large-scale integrated devices based on inorganic NWs: (1) selective growth of NWs, (2) selective assembly of pre-grown NWs, and (3) direct printing of NWs.

\subsection{Selective growth of inorganic nanowires}

One-dimensional crystal growth essentially exploits the highly asymmetric growth kinetics in the radial and axial directions. ${ }^{1,2}$ One of the earliest and prevailing examples for one-dimensional semiconductors is the metal-catalytic chemical-vapor growth of NWs. ${ }^{3,4}$ Therein, metal catalysts at the nanometer scale, i.e. metal nanoclusters, are employed for the catalytic decomposition of the vapor precursors, the dimensionally confined nucleation and the subsequent one-dimensional growth. ${ }^{5}$ One of the urgent technological breakthroughs for the practical applications of these bottom-up semiconductor NWs is integrated NW array growth in large areas with massive parallelism. In this section, we overview the recent developments of the NW growth, by which the size, position and direction of NWs are predictably controlled. Within the framework of catalytic chemical-vapor growth, this can be achievable by controlled NW growth from the ordered catalyst arrays with the deterministic one-to-one

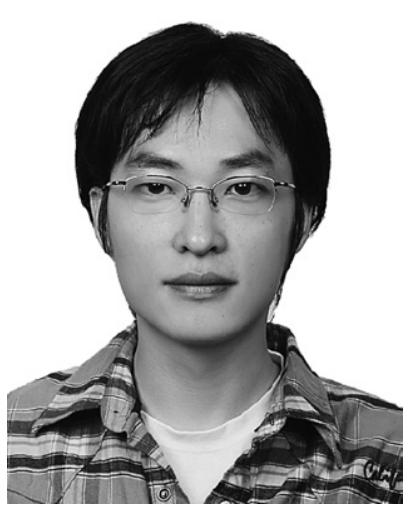

Kwang Heo
Kwang Heo received his B.S. degree in Materials Science and Engineering from Seoul National University in 2005. He is currently a Ph.D. candidate in the Interdisciplinary Program in Nano-Science and Technology at Seoul National University under the supervisor of Prof. Seunghun Hong. His current research topic is the massive assembly of inorganic nanowires on various substrates and device fabrication based on nano-materials.

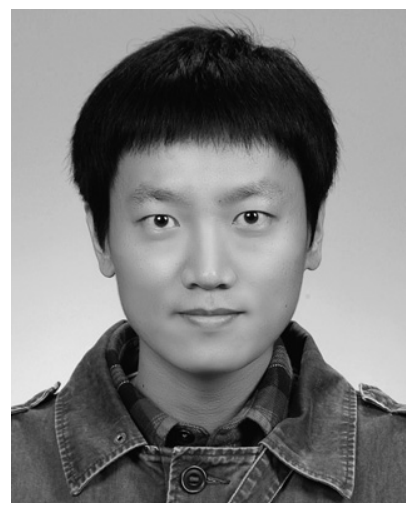

Cheol-Joo Kim
Cheol-Joo Kim earned his B.S. degree in Materials Science and Engineering from Pohang University of Science and Technology (POSTECH) in 2006. He is currently a Ph.D. candidate in Department of Materials Science and Engineering at POSTECH under the guidance of Prof. Moon-Ho Jo. His research interests include controlled growth of group IV semiconductor nanowires and their applications into optoelectronics. 
relation in size, position and direction between catalysts and NWs.

\subsection{Catalytic chemical-vapor growth of nanowires}

We begin our discussion with the different types of metal catalysts, which determine the subsequent NW growth behavior.

Conventionally metal nanoclusters are often involved in growth reactions as eutectic liquid catalysts by providing lower activation energy for crystallization. Hence the thermodynamic limit of such catalytic NW growth is largely set by the lowest eutectic temperature of the binary systems of metals and semiconductors; for example the lowest eutectic temperatures for (Au, Si) and $(\mathrm{Au}, \mathrm{Ge})$ binary systems were found to be around $360-370{ }^{\circ} \mathrm{C}$ in the bulk limit. In recent example, the catalysts are not necessarily the simple eutectic liquids during the growth reactions, but can also be solid phases. ${ }^{6}$

Fig. 1 (a) and (b) show transmission electron microscope (TEM) images of Au-catalyzed Ge NWs, where Au catalysts are involved in the growth reaction as eutectic liquids or solids depending on the growth temperature and thermal cycling history. These synthetic routes from the solid catalysts are evident by the accumulating examples of semiconductor-catalyst systems, such as $\mathrm{Si}-\mathrm{Ti},{ }^{7} \mathrm{Ge}-\mathrm{Ni},{ }^{8} \mathrm{GaAs}-\mathrm{Au},{ }^{9} \mathrm{InAs}-\mathrm{Au},{ }^{10} \mathrm{Si}-\mathrm{Al},{ }^{11} \mathrm{Si}-\mathrm{Cu},{ }^{12}$ $\mathrm{Mn}-\mathrm{Ge}^{13}$ and Ge-Au. ${ }^{14}$ Therein it has been commonly documented that the NW crystallization can occur by solid-phase diffusion of semiconductor elements through the solid catalysts, ${ }^{9}$ often at temperatures below the eutectic temperature. ${ }^{\mathbf{1 1 , 1 4}}$

\subsection{Shape (diameter and length) controlled NW growth by solid catalysts}

The one-to-one deterministic relation between catalysts and NWs may break down when the catalyst particles migrate along the surfaces of NWs and substrates, and sometimes coalescence during the NW growth, leading to unpredictable NW shape formation (Fig. 1 (c) and (d)). For example, Hannon et al. showed that during the Au-catalytic NW growth, Au is not spatially confined within the catalyst droplets and migrates to neighboring NWs by wetting the NW sidewalls, and eventually consuming the droplets and terminating catalytic growth as seen in Fig. 1 (c) and (d). ${ }^{15,16}$ Thermodynamically Au migrates from smaller droplets to larger ones, i.e. by an Ostwald ripening process, leading to non-uniform diameter distributions within an individual NW, and from NW to NW. The axial NW growth kinetics is also closely related to the NW diameters, and is believed to be strongly dependent on the specific rate limiting steps in the NW crystallization reactions. ${ }^{17,18,19}$

The diameter distribution in the NW array growth can be made more uniform by suppression of surface migration and coalescence using solid-phase catalysts. Fig. 1 (e) and (f) illustrate empirical manifestations in examples of Ge NW growth from $\mathrm{Au}$ nanoparticles, in direct comparison to the growth from $\mathrm{Cu}$ nanoparticles, where they represent liquid catalysts and solid catalysts in the thermodynamic limit, at $330{ }^{\circ} \mathrm{C}(\mathrm{Au}, \mathrm{Ge})$ and $275^{\circ} \mathrm{C}(\mathrm{Cu}, \mathrm{Ge})$, respectively. ${ }^{20}$ The diameters of Ge NWs from $\mathrm{Au}-\mathrm{Ge}$ eutectic liquids diverge in their distribution differently from the initial catalysts of $7 \mathrm{~nm}$ due to the grain growth up to $20 \mathrm{~nm}$. Meanwhile the $\mathrm{Cu}$ catalysts remain as individual grains with the mean diameter of $7 \mathrm{~nm}$ without any obvious grain growth at the growth temperature, and lead to growth with uniform diameters of $7 \mathrm{~nm}$. This finding provides a practical indication that the NW diameter distribution can be deterministically controllable for large-area integrations of NWs, when NWs grow by solid catalysts.

\subsection{Orientation controlled NW growth by epitaxy}

It is possible to apply conventional epitaxial thin film growth to catalytic NW growth to achieve orientational control of NWs in the arrays, assuming that all the constituent NWs possess identical crystal orientations. In fact the epitaxy between the NWs and the substrates can be energetically facilitated in the presence of catalysts in between them.

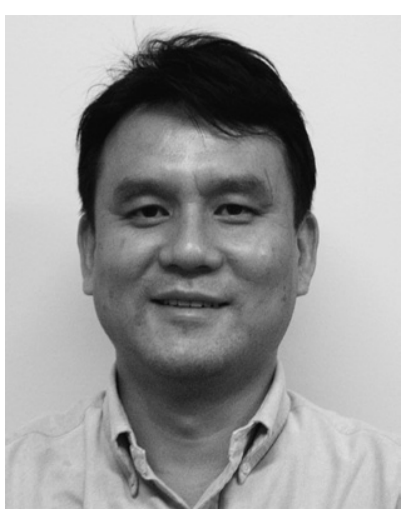

Moon-Ho Jo
Moon-Ho Jo received his Ph.D. in Materials Science at University of Cambridge (2001), with a dissertation on electron spin tunneling in half-metallic manganites. He joined the faculty of the Department of Materials Science and Engineering at POSTECH in 2004 after a three-year postdoctoral fellowship in the Department of Chemistry at Harvard University, where his research dealt with single-electron transport through individual molecules and other low-dimensional systems. His current research interests include gas-phase syntheses of semiconductor nanowires and their fundamental properties for electronics/photonics/photovoltaics applications.

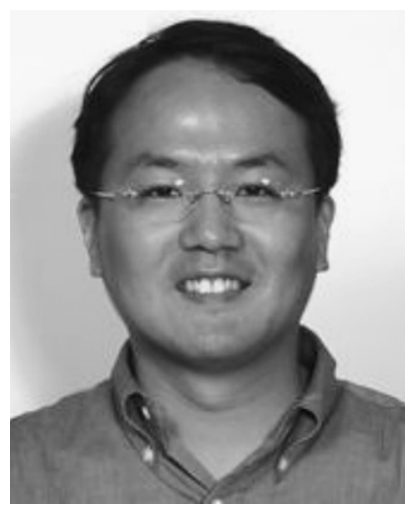

Seunghun Hong
Seunghun Hong finished his PhD in Physics (1998) at Purdue University. He worked as a postdoc and research professor in the Chemistry Department at Northwestern University from 1998 to 2001. He was an assistant professor in the Physics Department at Florida State University from 2001 to 2003. Since then, he has been working as an assistant (2003-2005) and associate (2006-now) professor in the Physics Department at Seoul National University. His research fields include molecular electronics, dip-pen nanolithography, self-assembly, surface-programmed assembly, CNT electronics, nano-biosensors, nanoscale tissue engineering, and biological motors. 

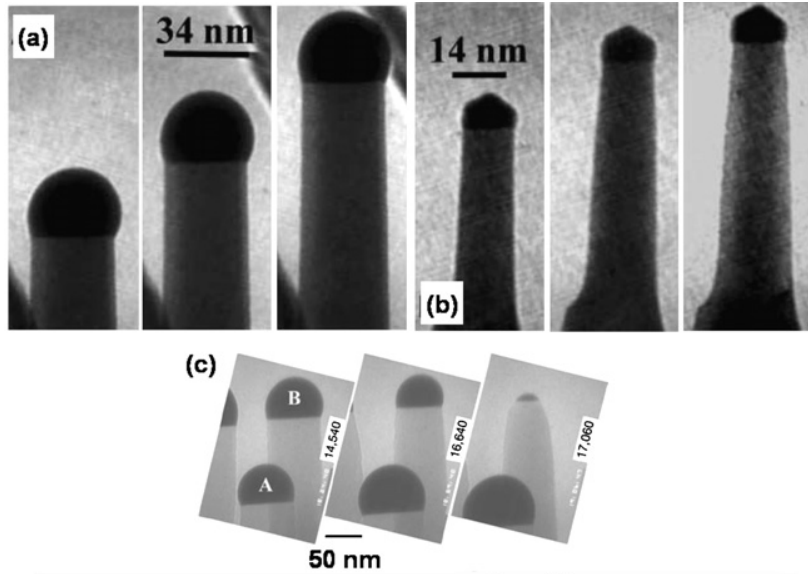

(d)
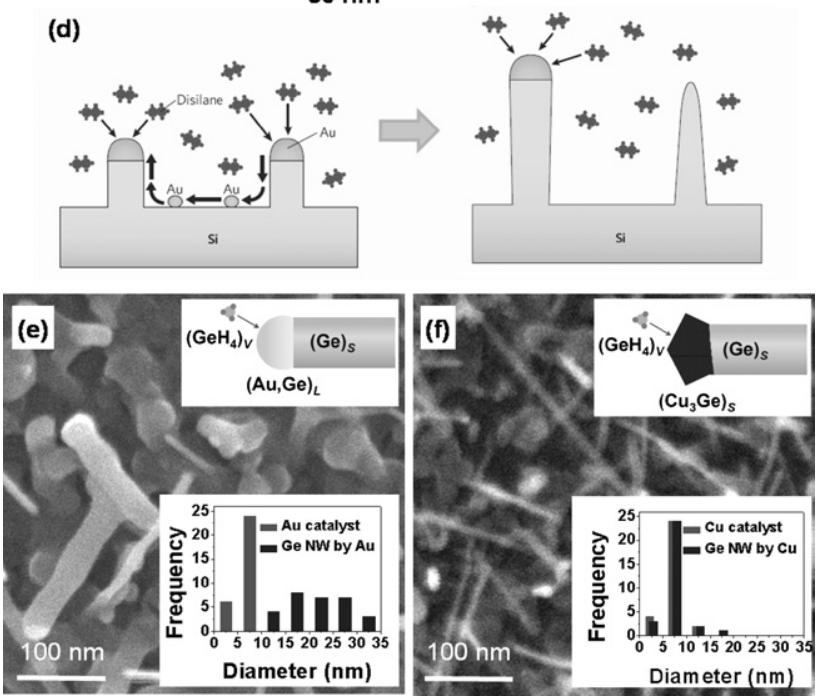

Fig. 1 (a) Series of images of a single Ge wire acquired at times $t=0$, 309 , and $618 \mathrm{~s}$ (from left to right, respectively) during growth using the liquid $\mathrm{Au} / \mathrm{Ge}$ alloy catalyst. (b) Another image series for a second wire growing at the same temperature and pressure but with a solid catalyst at $\mathrm{t}=0,1340$, and $1824 \mathrm{~s}$ (from left to right, respectively). (c) Three images labelled by the time (in seconds) after the start of Ge nanowire growth in ultra-high vacuum conditions. (d) Schematic of the Au diffusion process during the growth in (c). (e) Scanning electron microscopy (SEM) image after $30 \mathrm{~s} \mathrm{GeH}_{4}$ chemical vapor deposition (CVD) on $0.5 \mathrm{~nm}$ thick $\mathrm{Au}$ films. The upper inset shows the schematic of Au catalytic growth of $\mathrm{Ge}$ nanowires. The lower inset shows the histogram of the diameter distribution for Au catalysts and the resultant Ge nanowires. (f) The same informations as in (e) for the case of 2 min deposition of $\mathrm{GeH}_{4}$ on $0.5 \mathrm{~nm}$ thick $\mathrm{Cu}$ films. Images (a) and (b) are adapted from ref. 14, (c) from ref. 15 , (d) from ref. 16, and (e) and (f) from ref. 20.

Fig. 2 (a) shows the high resolution TEM image of an epitaxially grown $\mathrm{Si}$ NW on (111) Si substrates by Au catalysts. ${ }^{21}$ The axial crystallographic orientation of Si NWs is along the $<111>$ direction and this clearly shows the epitaxial relation between the substrate and the NW. Fig. 2 (b) shows the tilted view of vertically aligned $\mathrm{Si}$ NWs on (111) Si substrates grown by using solid-Al catalysts. ${ }^{11}$ Fig. 2 (c), (d), and (e) show the representative top view scanning electron microscopy (SEM) images of epitaxially grown Si nanowires on (111), (110), and (100) substrates, respectively. ${ }^{22,23}$ Lower insets show the crosssectional views, where the white arrow represents the $<111>$
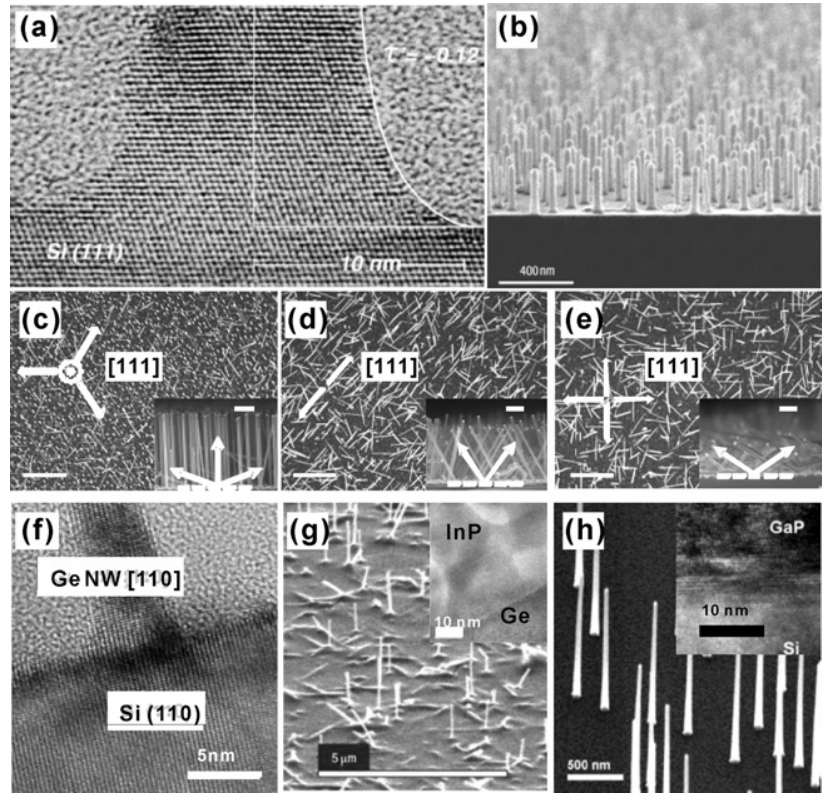

Fig. 2 (a) High resolution TEM of an epitaxially grown silicon nanowire on a $\mathrm{Si}$ (111) substrate. (b) SEM cross-sectional image (tilt $10^{\circ}$ ) of $\mathrm{Si}$ nanowires epitaxially grown using $\mathrm{Al}$ catalysts at $430^{\circ} \mathrm{C}$. (c)-(e) Top view images of Si nanowire array grown on (c) $\mathrm{Si}$ (111), (d) $\mathrm{Si}$ (110), and (e) $\mathrm{Si}$ (100) substrate. Insets show the cross-section view images. $<111>$ directions with respect to the crystal orientation of the substrate are indicated by arrows. Scale bars for the top view and cross-section view are $10 \mu \mathrm{m}$ and $2 \mu \mathrm{m}$, respectively. (f) High resolution TEM image of a vertically grown Ge NW on a $\mathrm{Si}$ (110) substrate. (g) SEM image of a sample with InP nanowires grown on $\mathrm{Ge}$ (111). Inset shows the high resolution TEM image of an InP wire on a Ge (111) substrate. (h) A $45^{\circ}$ tilt SEM micrograph of $\mathrm{GaP}$ nanowires growing vertically from the $\mathrm{Si}$ (111) surface in the [111] direction. Inset shows the high resolution TEM image of the $\mathrm{Si}$ substrate-GaP nanowire interface. The crystal directions from the Si substrate are transferred to the nanowire. Image (a) adapted from ref. 21, (b) from ref. 11, (c)-(e) from unpublished results from the present authors (Cheol-Joo Kim and Moon-Ho Jo), (f) from ref. 20, (g) from ref. 25 , and (h) from ref. 26.

orientation of Si NWs. This further shows that the growth orientation of Si NWs can be controlled by epitaxial NW growth on substrates with different crystal orientations.

Recently heteroepitaxial NW growth has been also demonstrated, as seen in Fig. 2(f)-(h), such as Ge NWs on $\mathrm{Si}$ substrates, ${ }^{20,24} \mathrm{InP}$ NWs on Ge substrates, ${ }^{25} \mathrm{GaAs}$ NWs on $\mathrm{Si}$ substrates, ${ }^{9} \mathrm{GaP}$ NWs on $\mathrm{Si}$ substrates, ${ }^{26} \mathrm{GaN}$ NWs on sapphire substrates ${ }^{27}$ and $\mathrm{ZnO} \mathrm{NWs}$ on sapphire substrates. ${ }^{28}$ It is reported that the finite size of NWs makes it easier to accommodate strain arising from the lattice mismatch between NWs and substrates, and thus homo- and heteroepitaxy in NW growth is easier to achieve than in the thin-film counterparts.

\subsection{Position controlled NW growth by deterministic growth}

Within the framework of catalytic NW growth, it is apparent that the NW positions in the arrays can be defined by the preparation of well-ordered catalyst arrays. Various nano-patterning techniques for large-area NW array pattern growth have been 
proposed to provide periodic arrangements with feature sizes and lattice constants below $100 \mathrm{~nm}$, such as atomic force microscope (AFM) lithography, ${ }^{29}$ electron-beam lithography (EBL), ${ }^{30}$ nanosphere lithography, ${ }^{31}$ nanoimprint lithography (NIL),${ }^{32}$ and other self-assembly templates including block copolymers and anodic aluminium oxides (AAO).$^{33}$

Such representative examples are presented in Fig. 3. Fig. 3 (a) shows aligned $\mathrm{ZnO} \mathrm{NW}$ arrays grown on a predesigned pattern by nanoindentation by AFM tips on PMMA films, followed by a lift-off for catalyst deposition. ${ }^{29}$ This technique allows a high degree of control in selectivity, nucleation density, and positioning accuracy, but requires too much time to fabricate for large area applications. In Fig. 3 (b), EBL and a metal lift-off method were used to pattern substrates with $\mathrm{Au}$ catalysts. ${ }^{30}$ Vertical InP NW arrays were then grown from the gold particles, using metal-organic vapor phase epitaxy. Chemical-solution based methods may provide an inexpensive process that is easily scalable to large-area applications and Fig. 3 (c) shows SEM images of a highly ordered honeycomb array of aligned carbon nanotubes grown by plasma-enhanced CVD from patterned catalysts by using a monolayer of polystyrene nanospheres as a negative mask for catalyst deposition, called nanosphere lithography. ${ }^{31}$ Fig. 3 (d) shows the SEM images of NIL defined NW arrays as obtained after growth on NIL-patterned Au catalysts. ${ }^{32}$ Growth of vertically aligned InP NWs was subsequently performed using a metal-organic vapor phase epitaxy technique. Reportedly NIL is in many respects capable of producing results comparable to those of EBL, but at a considerably lower cost and with a much higher throughput for largescale integration of NWs.
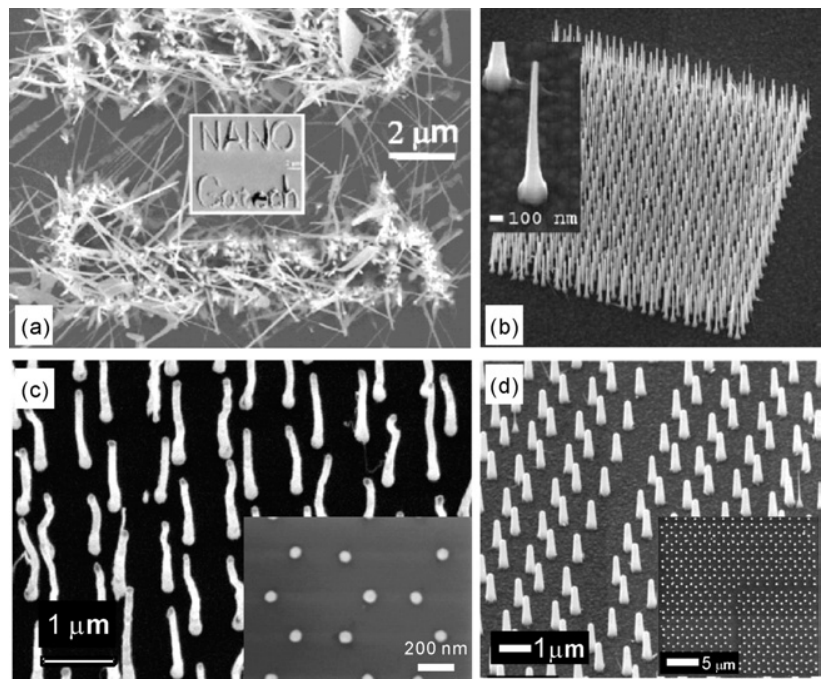

Fig. 3 (a) Complex patterns of $\mathrm{ZnO} \mathrm{NW}$ arrays by AFM lithography technique: "NANO Gatech" viewed from the top. The inset is a SEM image of the original Au pattern. (b) Vertical InP (111) nanowire arrays on e-beam patterned Au catalysts. (c) Inset shows the SEM image of highly ordered honeycomb array of aligned Ni dots patterned by nanosphere lithography. Main image shows the carbon nanotubes grown from the catalyst pattern. (d) SEM cross-sectional image (tilt $45^{\circ}$ ) of NIL-defined InP nanowire arrays. Inset shows the top view image. Image (a) adapted from ref. 29, (b) from ref. 30, (c) from ref. 31, and (d) from ref. 32.

\subsection{Selective assembly of nanowires in solution}

Although the chemical vapor deposition method allows one to prepare high-purity nanowires, it is often difficult to mass produce large amounts of NWs. On the other hand, some nanowires can be mass produced by solution-phase synthesis. For large scale integrated device applications, the NWs in solution should be selectively assembled onto the desired locations of the substrate with precise orientation. This section will discuss the selective assembly method of pre-grown inorganic nanowires.

\subsection{Solution-based chemical synthesis of nanowires}

Compared with vapor-phase growth process methods, solutionphase synthetic routes have various advantages such as relatively low temperatures, low cost, convenience in handling, and easiness in composition control. These advantages make them very promising for the large-scale synthesis of one dimensional (1D) nanostructures. Solution-based chemical synthesis of nanowires has several typical synthetic routes based on controlled precipitation from homogeneous solutions, including hydrothermal/ solvothermal processes, solution-liquid-solid (SLS) processes, solution-phase methods based on capping reagents, and lowtemperature aqueous-solution processes.

Hydrothermal/solvothermal synthesis utilizes a solvent under pressures and temperatures above its critical point to increase the solubility of a solid and to accelerate reactions between solids (Fig. 4 (a)). In a sealed vessel, solvents can be brought to
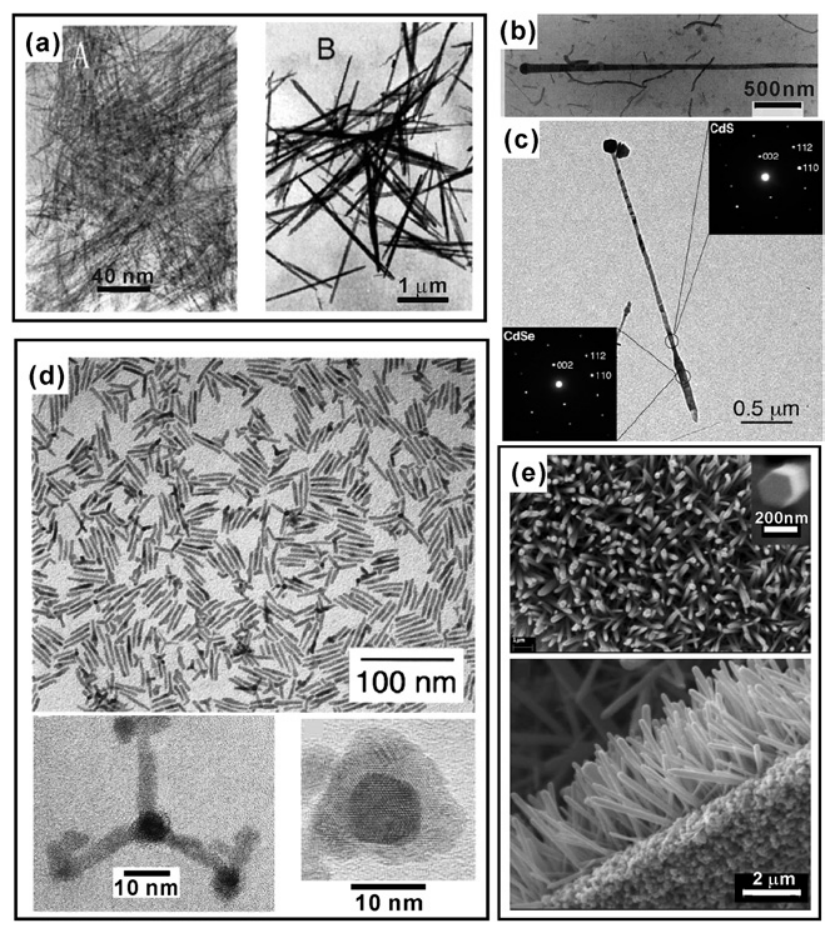

Fig. 4 Nanowires synthesized by solution-based chemical synthesis. (a) TEM images of $\alpha-\mathrm{MnO}_{2}$ and $\beta-\mathrm{MnO}_{2}$ by hydrothermal/solvothermal synthesis. (b) TEM image of InP nanowires by a solution-liquid-solid (SLS) growth process. (c) TEM image of Bi/CdS/CdSe nanorods by a SLS growth process. (d) TEM images of CdSe nanorods by solution-phase methods based on capping reagents. (e) FE-SEM images of $\mathrm{ZnO}$ nanorods grown by the aqueous chemical method. Image (a) adapted from ref. 35 , (b) from ref. 40, (c) from ref. 41, (d) from ref. 43, and (e) from ref. 47. 
temperatures well above their boiling points by increasing pressures resulting from heating. Heath and co-workers pioneered the use of solvothermal synthesis of semiconductor nanowires. ${ }^{34}$ They demonstrated the synthesis of single-crystalline Ge nanowires in an alkane solvent heated to $275^{\circ} \mathrm{C}$ with a pressure of 100 atm. This method was later exploited extensively by Quan, Xie, Lie and co-workers to synthesize various structures such as wires, ${ }^{35}$ tubes ${ }^{36,37}$ and whiskers. ${ }^{38,39}$

Since it was first reported in 1995, the solution-liquid-solid (SLS) growth process has been a very useful method to prepare 1D semiconductor nanostructures (Fig. 4 (b) and (c)). The SLS growth process needs molten metal liquid droplets as a catalyst to guide the growth of $1 \mathrm{D}$ nanostructures. In the SLS growth process, the organic solution containing reactive precursors and metal nanocrystal catalysts is heated to a relatively high temperature. Then, liquid droplets which catalyze the decomposition of the precursors are formed by melting metal catalysts. The yielded species are dissolved into the metal liquid droplets until supersaturation is reached, then they are separated out to form 1D nanostructures. Buhro and co-workers developed a SLS method for the synthesis of high crystalline nanowires of III-V semiconductors at relatively low temperature. ${ }^{40}$ In addition, complex architectures of 1D semiconductor nanostructures can also be fabricated by the SLS growth process. Recently, novel $\mathrm{CdS} / \mathrm{CdSe}$ nanorod heterostructures were synthesized through SLS growth mechanism. ${ }^{41}$ These heterostructures were synthesized by controlling sequences of CdS and CdSe materials. This result shows that SLS synthesis provides a very efficient process to prepare axial nanowire heterostructures that were difficult to synthesize using either VLS growth or catalyst-free solution-phase synthesis.

1D semiconductor nanostructures can also be synthesized in solutions with suitable capping agents or surfactants through kinetically controlled anisotropic growth or oriented attachment (Fig. 4 (d)). The growth mechanism of this process is explained by the growth kinetics of the crystal shape. The slowest growing planes as the facets of the product are left behind while the fastest growing planes disappear. The difference of growth kinetic velocity is more extensively controlled by appropriate capping reagents. Utilizing appropriate capping reagents, the final shape of a crystal is controlled by changing the free energies of the various crystallographic surfaces. In this aspect, it has turned out to be a very effective method in the fabrication of high-quality 1D semiconductor nanostructures and more complex nanoarchitectures. Utilizing this approach, Alivisatos and co-workers first demonstrated controlling the shape of CdSe nanocrystals by using mixed surfactants. ${ }^{42,43}$ They synthesized complex structures, such as tetrapod-shaped, arrow-shaped and pine treeshaped CdSe nanocrystals, as well as CdSe nanorods. Xia and co-workers demonstrated the polyol method to generate silver nanowires by reducing silver nitrate with ethylene glycol in the presence of poly(vinyl pyrrolidone) (PVP). ${ }^{44,45}$ In addition, 1D nanostructures are synthesized in solution without surfactants by the intrinsically anisotropic crystallographic structure of a semiconductor material. Chalcogens of the trigonal phase such as $\mathrm{Se}$ and Te are suitable materials for synthesizing 1D nanostructures. By Mayers and Xia, it was reported that tellurium nanotubes could be synthesized by adding orthotelluric acid to pure ethylene glycol refluxed at $197{ }^{\circ} \mathrm{C}^{46}$ In this process, ethylene glycol could serve as both solvent and reducing reagent.
However, these synthetic approaches usually require relatively high temperature and organic solvents in many cases (Fig. 4 (e)). It would be highly desirable to develop facile synthesis of $1 \mathrm{D}$ semiconductor nanostructures in aqueous solutions at relatively low temperature. Vayssieres first reported that two-dimensional arrays of $\mathrm{ZnO}$ nanorods could be obtained by placing various substrates in an aqueous solution containing zinc nitrate and methenamine and heating the reaction system to $95^{\circ} \mathrm{C}$ for several hours in a regular laboratory oven. ${ }^{47}$ This process is a very simple, one-step, aqueous, low-temperature growth process for the inexpensive fabrication of large area ordered crystalline $\mathrm{ZnO}$ nanorods without the need for template, surfactant, applied field, or undercoating on various substrates. Vanadium pentoxide $\left(\mathrm{V}_{2} \mathrm{O}_{5}\right)$ nanowires are also obtained by polycondensation of vanadic acid in water. ${ }^{48}$

\subsection{Directed assembly of nanowire-based integrated devices}

Applied electric fields (e-field) can be used to effectively align NWs due to their highly anisotropic structures and large polarization (Fig. 5 (a)). ${ }^{49}$ NWs can be easily polarized in an alternating electric field due to charge separation at the surface of NWs. Smith et al. ${ }^{49}$ demonstrated the e-field assisted assembly of metallic NWs. They showed the assembly process was dependent on the applied voltage and frequency, indicating that the alignment forces resulted from the polarization of the NWs in the alternating electric field. The electric-field assembly method can also be used to assemble individual NWs into parallel and crossed arrays. ${ }^{50}$ Electric-field assembly is suitable for organizing individual NWs with good directional and spatial control.

Fluidic-flow directed assembly is also a powerful approach for aligning nanowires (Fig. 5 (b)). ${ }^{51}$ In this method, a fluidic channel structure made of PDMS is in contact with a flat substrate with surface molecular patterns. When passing the NW suspensions through the fluidic channel structures, NWs are assembled onto the molecular patterns, and they are aligned along the flow direction. This fluidic approach can also be used to assemble crossed arrays and geometrically more complex structures by simply controlling the angles of flow directions.

The Langmuir-Blodgett (L-B) technique can be utilized for the assembly of NWs with large aspect ratios (Fig. 5 (c)). For example, the L-B technique has been utilized to align silicon and silver NWs. ${ }^{52,53}$ In this method, the NWs are first floated on the surface of water. Then, the floating NWs are compressed to higher density using barriers. This layer of aligned NWs can be transferred onto any substrate for the fabrication of devices based on aligned NWs. In addition, crossed NW structures and more hierarchical NW building blocks can be organized by transferring to planar substrates in a layer-by-layer process. ${ }^{54}$

The directed assembly method using receptor-mediated interactions provides a powerful tool for the site specific assembly of NWs and complex architectures based on NWs (Fig. 5 (d)). Salem et al. applied the receptor-mediated assembly strategy to position a large number of multicomponent nanowires. ${ }^{55}$ In this work, two segment $\mathrm{Au} / \mathrm{Ni} \mathrm{NW}$ were anchored to each other using biotin-avidin linkages. This biotin-avidin linkage is one of the strongest linkers, which form well-known biological interactions with stability over a broad $\mathrm{pH}$ range. They demonstrated the assembly of multifunctional NWs in spatially localized 


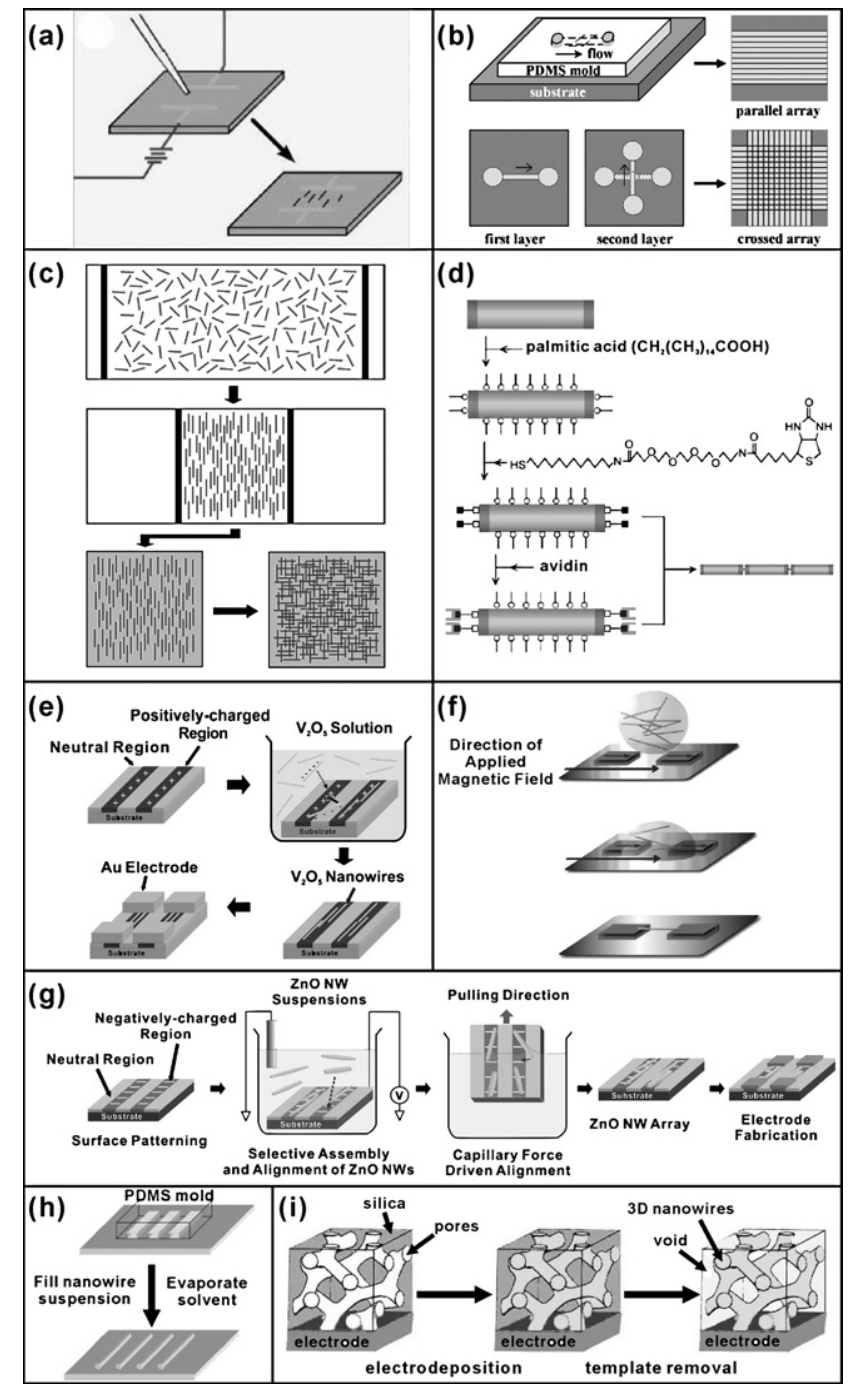

Fig. 5 Schematic diagram depicting directed assembly of nanowirebased integrated devices. (a) Electric field assembly. (b) Fluidic flow assembly. (c) Langmuir-Blodgett technique. (d) Receptor-mediated assembly. (e) Surface-programmed assembly. (f) Magnetic field assembly. (g) Capillary force assembly. (h) Microfluidic-assisted nanowire integration (MANI) process. (i) Templated electrodeposition technique. Image (a) adapted from ref. 50, (b) from ref. 51, (c) from ref. 54, (d) from ref. 56, (e) from ref. 57, (f) from ref. 58, (g) from ref. 60, (h) from ref. 61 and (i) from ref. 62.

regions with selective surface functionalization. In addition, Chen et al. showed directed end to end assembly of multisegment NWs using biotin-avidin linkages. ${ }^{56}$

Surface-programmed assembly is an effective process to assemble a large number of NWs using only conventional microfabrication processes. In this process, self-assembled monolayer (SAM) patterns were prepared via a conventional microfabrication process, and they were used to direct the adsorption and alignment of NWs on solid substrates in solution. Myung et al. applied the surface-programmed assembly strategy to assemble pristine $\mathrm{V}_{2} \mathrm{O}_{5} \mathrm{NWs}$ with high precision on solid substrates (Fig. 5 (e))..$^{57}$ In this work, the substrates were first patterned with SAM molecules to create positively charged and neutral surface regions. On Au surfaces, cysteamine and ODT were utilized to generate positively charged and neutral SAMs, respectively. APTES and OTS were patterned to create positively charged and neutral regions on $\mathrm{SiO}_{2}$ surfaces. When the substrate was placed in the aqueous solution of $\mathrm{V}_{2} \mathrm{O}_{5} \mathrm{NWs}$, the NWs were attracted to positively charged regions by electrostatic forces because the $\mathrm{V}_{2} \mathrm{O}_{5} \mathrm{NWs}$ were usually charged negatively in water. Since the adsorbed NWs form stable structures, one can continue additional microfabrication steps to fabricate functional devices such as FETs.

Magnetic force-driven self-assembly process on magnetic templates is a sophisticated technique, relying on an intrinsic energy source, for aligning and positioning ferromagnetic NWs such as nickel, cobalt, and permalloy (Fig. 5(f)). Yoo et al. demonstrated the manipulation and positioning of ferromagnetic NWs on pre-patterned ferromagnetic electrodes. ${ }^{58}$ In this work, suspensions of Ni NWs were dispensed on the top of the ferromagnetic electrodes. The NWs were attracted to the electrodes by the magnetic force, leading to the adsorption of the NWs to the electrodes. In addition, the direction of assembled NWs can be controlled by changing the external field direction. ${ }^{59}$

Capillary forces are also very efficient forces for controlling the assembly and alignment of NWs (Fig. 5(g)). Kang et al. demonstrated assembly of $\mathrm{ZnO} \mathrm{NWs}$ using capillary forces combined with a surface programmed assembly process.$^{60}$ In this work, $\mathrm{ZnO}$ NWs were first adsorbed onto the negatively charged SAM patterns in solution. Then, the adsorbed NWs were further aligned along a specific direction by pulling out the substrate from the solution along the desired direction. In this process, the strong surface tension of water was exerted on the adsorbed $\mathrm{ZnO}$ NWs, resulting in improved alignment.

Hierarchical structures of nanowire building blocks have been fabricated by utilizing templates such as PDMS molds (Fig. 5(h)), ${ }^{61}$ mesoporous structures (Fig. 5(i) $)^{62}$ and block-copolymer templates. ${ }^{63}$ Messer et al. demonstrated the microfluidicassisted nanowire integration (MANI) process (Fig. 5(h)). ${ }^{64} \mathrm{In}$ this method, desired structures of nanowires were prepared by filling the microchannels with the nanowire suspension and evaporating the solvent. This technique had been successfully applied for the alignment of $\mathrm{Mo}_{3} \mathrm{Se}_{3}{ }^{-}$molecular wires. Yunfeng Lu's group also fabricated hierarchical structures of nanowires based on a templated electrodeposition process (Fig. 5(i)). ${ }^{62,65}$ They created a replicated mesoporous nanowire network by filling with metals or semiconductors into the mesoporous silica film and removing the silica template. These hierarchical nanowire structures have potential for various applications such as sensors, catalysts, fuel cells and photovoltaics.

\subsection{Direct printing of nanowires}

Dry transfer printing provides a very efficient approach for assembling nanostructures and fabricating multilayer building block-based nanostructures (Fig. 6 (a)). ${ }^{6-68}$ In this method, rubber stamps are utilized to pick up nanostructures from the source substrate and to drop them off onto the target substrates. Various strategies have been developed to enhance the success rate of these pick-up and drop-off processes. One strategy is relying on viscoelastic properties of rubber stamps. In this method, desired micro- or nanostructures are first prepared on solid substrates via conventional microfabrication or growth 


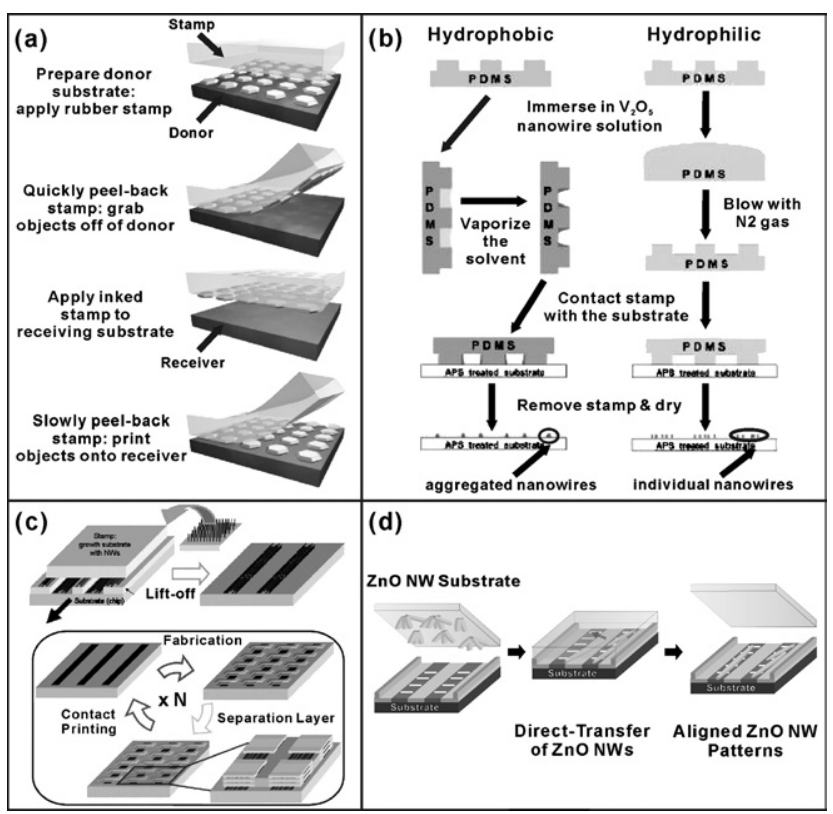

Fig. 6 Schematic illustrations of printing of nanowire-based integrated devices. (a) Dry-transfer printing. (b) Microcontact printing. (c) Direct contact printing. (d) Direct transfer printing. Image (a) adapted from ref. 68, (b) from ref. 70, (c) from ref. 72, and (d) from ref. 60.

methods. Then, a rubber stamp makes contact with the source substrate and is detached rather rapidly. The strength of adhesion between the stamp and the nanostructure is rate dependent due to the viscoelastic properties of the stamp. At a slow detachment speed, the energy required to separate the stamp from the substrate is relatively low, because of the low surface energy of the stamp. At fast speeds, however, the energy required to separate is much greater. Thus, by detaching the stamp from the source substrate with a fast detachment speed, the nanostructures can be transferred from the source substrate to the stamp. Finally, the nanostructures on the stamp can be transferred to the desired target substrates by applying the stamp onto the target substrate and detaching it slowly.

The micro-contact printing (MCP) method was first developed by Whitesides' group to deposit organic molecules, and it has been widely used to stamp organic and inorganic substrates onto solid substrates. ${ }^{69} \mathrm{Kim}$ et al. had directly transferred $\mathrm{V}_{2} \mathrm{O}_{5} \mathrm{NW}$ patterns onto solid substrates using the MCP technique (Fig. 6 (b)). ${ }^{70}$ In the work, they used hydrophobic and hydrophilic stamps to directly transfer $\mathrm{V}_{2} \mathrm{O}_{5} \mathrm{NWs}$ onto substrates via different transfer mechanisms. In the case of hydrophobic stamps, the aqueous solutions of negatively charged $\mathrm{V}_{2} \mathrm{O}_{5} \mathrm{NWs}$ do not wet the relief sides but are stored in the recess regions of the stamp. Thus, $\mathrm{V}_{2} \mathrm{O}_{5} \mathrm{NWs}$ were transferred to the substrate along the edge of the stamp patterns. $\mathrm{V}_{2} \mathrm{O}_{5} \mathrm{NWs}$ were also transferred to the substrate through the relief side of the stamp using relatively hydrophilic stamps treated with hydrochloric acid.

For wafer-scale assembly and 3D multi-layer structures, a simple direct contact printing process is a very useful method (Fig. 6 (c)). ${ }^{71}$ This method was first discovered by Lieber's group. In this process, NWs are first grown vertically on solid substrates. The substrates with vertically grown NWs are then in contact with the receiver substrate and slide along a specific direction. During the process, NWs are effectively detached from the donor substrate as they are absorbed by the van der Waals interactions with the surface of the receiver substrate. As a result, NWs are aligned on the receiver substrate. Javey et al. applied the NW printing method and device fabrication steps were repeated multiple times in order to obtain vertically stacked electronic layers. ${ }^{72}$ To extend this process as a generic approach for scalable and large area printing, Yerushalmi et al. showed assembly of highly ordered arrays of NWs with high uniformity and reproducibility using differential roll printing. ${ }^{73}$

The direct-transfer method is a very efficient process to assemble the small amount of NWs synthesized on solid substrates for device applications. ${ }^{60}$ Kang et al. showed the assembly of $\mathrm{ZnO} \mathrm{NWs}$ utilizing this method. In this process, a ZnO NW film grown on a substrate was placed in close proximity to the receiver substrate with a SAM pattern. Then, ultrasonic vibration was applied so that NWs were detached and adsorbed onto the specific regions on the receiver substrate. During this process, $\mathrm{ZnO}$ NWs slide to minimize the interface energies between the NWs and SAM patterns so that one can achieve highly aligned NW patterns. Significantly, since this method enables the transfer of NWs without preparation of a NW solution, this process should be an ideal method for the assembly of small amounts of NWs grown on solid substrates for integrated device applications.

\subsection{Summary}

The invention of NW-based advanced devices resulted in a new paradigm in manufacturing functional devices. Unlike conventional microelectronics, one has to selectively grow NWs or assemble pre-grown NWs onto a specific location of the substrate with precise alignment for device fabrication. Promising strategies to solve this problem include selective growth, selective assembly, and direct printing. These methods complement each other for specific applications and may pave the way toward industrial applications of new advanced devices based on inorganic NWs.

However, several challenging problems should be solved by future research for practical applications of nanowire-based devices. One example of such a problem is the integration of a nanowire-assembly process with conventional semiconductor processes in industry since many nanowire-assembly methods (including some in this manuscript) require unconventional equipment or harsh processing steps which are not compatible with conventional microfabrication processes. Another problem might be reliable massive production of high quality nanowires. Until now, only limited research groups in the world have access to high quality nanowires, which has been significantly slowing the progress of nanowire-based device research.

\section{Acknowledgements}

SH acknowledges the support from the NRL (No. R0A-2004000-10438-0) program of KOSEF and the partial support from the Next Generation New Technology Development program of MKE and the NBIT program of KICOS. CJK and MHJ acknowledge the financial support by Nano R\&D program 
through the KOSEF (2007-02864), the "System IC 2010" program by the MOCIE, and Samsung Electronics.

\section{References}

1 L. Manna, D. J. Milliron, A. Meisel, E. C. Scher and A. P. Alivisatos, Nat. Mater., 2003, 2, 382.

2 Y. Xia, P. Yang, Y. Sun, Y. Wu, B. Mayers, B. Gates, Y. Yin, F. Kim and H. Yan, Adv. Mater., 2003, 15, 353.

3 R. S. Wagner and W. C. Ellis, Appl. Phys. Lett., 1964, 4, 89.

4 A. M. Morales and C. M. Lieber, Science, 1998, 279, 208.

5 Y. Wu and P. Yang, J. Am. Chem. Soc., 2001, 123, 3165.

6 V. Schmidt and U. Gosele, Sience, 2007, 316, 698.

7 T. I. Kamins, R. S. Williams, D. P. Basile, T. Hesjedal and J. S. Harris, J. Appl. Phys., 2001, 89, 1008.

8 H. Y. Tuan, D. C. Lee, T. Hanrath and B. A. Korgel, Chem. Mater., 2005, 17, 5705.

9 A. I. Persson, M. W. Larsson, S. Stenstrom, B. J. Ohlsson, L. Samuelson and L. R. Wallenberg, Nat. Mater., 2004, 3, 677.

10 S. G. Ihn and J. I. Song, Nanotechnology, 2007, 18, 355603.

11 Y. Wang, V. Schmidt, S. Senz and U. Gosele, Nat. Nanotech., 2006, 1, 186.

12 J. Arbiol, B. Kalache, P. R. Cabarrocas, J. R. Morante and A. F. Morral, Nanotechnology, 2007, 18, 305606.

13 J. L. Lensch-Falk, E. R. Hemesath, F. J. Lopez and L. J. Lauhon, J. Am. Chem. Soc., 2007, 129, 10670.

14 S. Kodambaka, J. Tersoff, M. C. Reuter and F. M. Ross, Science, 2007, 316, 729.

15 J. B. Hannon, S. Kodambaka, K. M. Ross and R. M. Tromp, Nature, 2006, 440, 69.

16 U. Gösele, Nature, 2006, 440, 34.

17 V. Schmidt, S. Senz and U. Gösele, Phys. Rev. B, 2007, 75, 045335.

18 S. Kodambaka, J. Tersoff, M. C. Reuter and F. M. Ross, Phys. Rev. Lett., 2006, 96, 096105.

19 M. T. Borgstrom, G. Immink, B. Ketelaars, R. Algra and E. P. A. M. Bakkers, Nat. Nanotech., 2007, 2, 541.

20 K. Kang, D. A. Kim, H. S. Lee, C. J. Kim, J. E. Yang and M. H. Jo, Adv. Mater., 2008, 20, 4684.

21 V. Schmidt, S. Senz and U. Gosele, Appl. Phys. A, 2005, 80, 445.

22 C. B Jin, J. E. Yang and M. H. Jo, Appl. Phys. Lett., 2006, 88, 193105.

23 J. E. Yang, C. B. Jin, C. J Kim and M. H. Jo, Nano Lett., 2006, 6, 2679.

24 T. I. Kamins, X. Li, R. S. Williams and X. Liu, Nano Lett., 2004, 4, 503.

25 E. P. A. M. Bakkers, J. A. V. Dam, S. D. Franceschi, L. P. Kouwenhoven, M. Kaiser, M. Verheijen, H. Wondergem and P. V. D. Sluis, Nat. Mater., 2004, 3, 769.

26 T. Martensson, C. P. T. Svensson, B. A. Wacaser, M. W. Larsson, W. Seifert, K. Deppert, A. Gustafsson, L. R. Wallenberg and L. Samuelson, Nano Lett., 2004, 4, 1987.

27 Z. Zhong, F. Qian, D. Wang and C. M. Lieber, Nano Lett., 2003, 3, 343.

28 M. H. Huang, S. Mao, H. Feick, H. Yan, Y. Wu, H. Kind, E. Weber, R. Russo and P. Yang, Science, 2001, 292, 1897.

29 J. H. He, J. H. Hsu, C. W. Wang, H. N. Lin, L. J. Chen and Z. L. Wang, J. Phys. Chem. B, 2006, 110, 50.

30 T. Martensson, M. Borgstrom, W. Seifert, B. J. Ohlsson and L. Samuelson, Nanotechnology, 2003, 14, 1255.

31 K. Kempa, B. Kimball, J. Rybczynski, Z. P. Huang, P. F. Wu, D. Steeves, M. Sennett, M. Giersig, D. V. G. L. N. Rao, D. L. Carnahan, D. Z. Wang, J. Y. Lao, W. Z. Li and Z. F. Ren, Nano Lett., 2003, 3, 13.

32 T. Martensson, P. Carlberg, M. Borgstrom, L. Montelius, W. Seifert and L. Samuelson, Nano Lett., 2004, 4, 699.

33 Z. H. Wu, X. Y. Mei, D. Kim, M. Blumin and H. E. Ruda, Appl. Phys. Lett., 2002, 81, 5177.

34 J. R. Heath and F. K. Legoues, Chem. Phys. Lett., 1993, 208, 263.

35 X. Wang and Y. Li, J. Am. Chem. Soc., 2002, 124, 2880.
36 Q. Lu, F. Gao and D. Zhao, Nano Lett., 2002, 2, 725.

37 T. Kasuga, M. Hiramatsu, A. Hoson, T. Sekino and K. Niihara, Langmuir, 1998, 14, 3160.

38 Y. Li, M. Sui, Y. Ding, G. Zhang, J. Zhuang and C. Wang, Adv. Mater., 2000, 12, 818.

39 Y. Xie, P. Yan, J. Lu, W. Wang and Y. Qian, Chem. Mater., 1999, 11, 2619.

40 T. J. Trentler, K. M. Hickman, S. C. Geol, A. M. Viano, P. C. Gibbons and W. E. Buhro, Science, 1995, 270, 1791.

41 L. Ouyang, K. N. Maher, C. L. Yu, J. McCarty and H. Park, J. Am. Chem. Soc., 2007, 129, 133.

42 X. G. Peng, L. Manna, W. D. Yang, J. Wickham, E. Scher, A. Kadavanich and A. P. Alivisatos, Nature, 2000, 404, 59.

43 L. Manna, E. C. Scher and A. P. Alivisatos, J. Am. Chem. Soc., 2000, 122, 12700.

44 Y. Sun, B. Gates, B. Mayers and Y. Xia, Nano Lett., 2002, 2, 165.

45 Y. Sun and Y. Xia, Adv. Mater., 2002, 14, 833.

46 B. Mayers and Y. Xia, Adv. Mater., 2002, 14, 279.

47 L. Vayssieres, Adv. Mater., 2003, 15, 464.

48 Jörg Muster, Gyu Tae Kim, Vojislav Krstic, Jin Gyu Park, Yung Woo Park, Siegmar Roth and Marko Burghard, Adv. Mater., 2000, 12, 420 .

49 P. A. Smith, C. D. Nordquist, T. N. Jackson, T. S. Mayer, B. R. Martin, J. Mbindyo and T. E. Mallouk, Appl. Phys. Lett., 2000, 77, 1399.

50 X. F. Duan, Y. Huang, Y. Cui, J. F. Wang and C. M. Lieber, Nature, 2001, 409, 66.

51 Y. Huang, X. F. Duan, Q. Q. Wei and C. M. Lieber, Science, 2001, 291, 630

52 D. Whang, S. Jin and C. M. Lieber, Nano Lett., 2003, 3, 951.

53 A. Tao, F. Kim, C. Hess, J. Goldberger, R. R. He, Y. G. Sun, Y. N. Xia and P. D. Yang, Nano Lett., 2003, 3, 1229.

54 D. Whang, S. Jin, Y. Wu and C. M. Lieber, Nano Lett., 2003, 3, 1255.

55 A. K. Salem, J. Chao, K. W. Leong and P. C. Searson, Adv. Mater., 2004, 16, 268

56 M. Chen and P. C. Searson, Adv. Mater., 2005, 17, 2765.

57 S. Myung, M. Lee, G. T. Kim, J. S. Ha and S. Hong, Adv. Mater., $2005,17,2361$.

58 B. Y. Yoo, Y. W. Rheem, W. P. Beyermann and N. V. Myung, Nanotechnology, 2006, 17, 2512.

59 C. M. Hangarter, Y. Rheem, B. Yoo, E. H. Yang and N. V. Myung, Nanotechnology, 2007, 18, 205305.

60 J. Kang, S. Myung, B. Kim, D. Oh, G. T. Kim and S. Hong, Nanotechnology, 2008, 19, 095303.

61 Y. Wu, H. Yan, M. Huang, B. Messer, J. H. Song and P. Yang, Chem. Eur. J., 2002, 8, 1261.

62 D. Wang, H. Luo, R. Kou, M. P. Gil, S. Xiao, V. O. Golub, Z. Yang, C. J. Brinker and Y. Lu, Angew. Chem. Int. Ed., 2004, 43, 6169.

63 J. Chai, D. Wang, X. Fan and J. M. Buriak, Nat. Nanotech., 2007, 2, 500.

64 B. Messer, J. H. Song and P. Yang, J. Am. Chem. Soc., 2000, 122, 10232.

65 D. Wang, H. P. Jakobson, R. Kou, J. Tang, R. Z. Fineman, D. Yu and Y. Lu, Chem. Mater., 2006, 18, 4231.

66 E. Menard, K. J. Lee, D. Y. Khang, R. G. Nuzzo and J. A. Rogers, Appl. Phys. Lett., 2004, 84, 5398.

67 Y. G. Sun and J. A. Rogers, Nano Lett., 2004, 4, 1953.

68 M. A. Meitl, Z. T. Zhu, V. Kumar, K. J. Lee, X. Feng, Y. Y. Huang, I. Adesida, R. G. Nuzzo and J. A. Rogers, Nat. Mater., 2006, 5, 33.

69 Y. Xia and G. M. Whitesides, J. Am. Chem. Soc., 1995, 117, 3274.

70 Y. K. Kim, S. J. Park, J. P. Koo, D. J. Oh, G. T. Kim, S. Hong and J. S. Ha, Nanotechnology, 2006, 17, 1375.

71 Z. Y. Fan, J. C. Ho, Z. A. Jacobson, R. Yerushalmi, R. L. Alley, H. Razavi and A. Javey, Nano Lett., 2008, 8, 20.

72 A. Javey, S. Nam, R. S. Friedman, H. Yan and C. M. Lieber, Nano Lett., 2007, 7, 773.

73 R. Yerushalmi, Z. A. Jacobson, J. C. Ho, Z. Fan and A. Javey, Appl. Phys. Lett., 2007, 91, 203104. 